Check for updates

Cite this: RSC Adv., 2019, 9, 3669

Received 16th December 2018 Accepted 22nd January 2019

DOI: $10.1039 / c 8 r a 10308 a$

rsc.li/rsc-advances

\section{The vivo antioxidant activity of self-made aged garlic extract on the D-galactose-induced mice and its mechanism research via gene chip analysis}

\begin{abstract}
Xiaomin Wang, (D) $\dagger^{\mathrm{ab}}$ Yukun Yang $\dagger^{\mathrm{c}}$ and Min Zhang ${ }^{* \mathrm{~b}}$
Two self-made aged garlic extract (AGE) were prepared and they were subjected with D-galactose-induced mice to explore vivo antioxidant effects and its mechanism via gene chip analysis. The biochemical analysis results indicated that AGE could significantly reduce the malondialdehyde (MDA) and lipofuscin content and increase the total superoxide dismutase (T-SOD), glutathione peroxidase (GSH-Px), catalase (CAT) activity. Histopathological observations found that AGE could improve the size, shape and arrangement state of liver and brain cells. Furthermore, gene expression profile array was used to screen 35 and 13 differentially expressed genes in liver and brain, respectively. Further analysis showed that the AGE could protect the mice from D-galactose-caused injury via carbohydrate metabolism, immunomodulatory, lipid metabolism, cell cycle regulation, amino acid metabolism and nervous regulation pathways. Through this experiment, we could comprehensively study the antioxidant mechanism of AGE and link the antioxidant function of AGE to the metabolic pathways.
\end{abstract}

\section{Introduction}

The free radical theory of aging states that in the aging process of aerobic metabolism, the increase of free radical levels or the lack of antioxidants will reduce the antioxidant capacity of the body, and thus increase the cytotoxicity, eventually leading to the irreversible damage of biofilms, amino acid and DNA, and accelerate the aging process. ${ }^{1}$ Antioxidants are reducing agents, which limit oxidative damage of biological structures by passivating them from free radicals. ${ }^{2}$ Nowadays, lots of reports have proved that antioxidants owns preventive and therapeutic effects on various chronic and degenerative diseases such as diabetes, ${ }^{3}$ neurodegeneration ${ }^{4}$ and cardiovascular disease, ${ }^{5}$ which are involved in the process of aging. ${ }^{6}$ Traditional synthetic antioxidants such as tert-butyl-p-hydroxyanisole (BHA) and dibutylhydroxytoluene (BHT) may be potentially toxic and harmful to humans. Therefore, as the trend of population aging is aggravating, growing attention of anti-aging has been given to natural products that are characterized as the antioxidant agents.

Aged garlic extract (AGE) is a garlic product formulated by soaking sliced raw garlic in low concentration aqueous ethanol

\footnotetext{
${ }^{a}$ Institute of Pharmaceutical and Food Engineering, Shanxi University of Chinese Medicine, Jinzhong 030619, Shanxi, China

${ }^{b}$ State Key Laboratory of Nutrition and Safety (Tianjin University of Science \& Technology), Tianjin 300457, China. E-mail: zm0102@tust.edu.cn; Fax: +86 22 60601445; Tel: +862260601445

'School of Life Science, Shanxi University, Taiyuan 030006, Shanxi, China

$\dagger$ These authors contribute equally to this work.
}

for up to 10 or 20 months at room temperature. ${ }^{7}$ In comparison with fresh garlic, AGE is odorless, and exhibits various biological activities, for instance, antioxidant, ${ }^{8}$ anti-atherosclerotic, ${ }^{9}$ anti-diabetic, anti-mutagenic, anti-carcinogenic, immunomodulation, ${ }^{10}$ cardioprotective, ${ }^{11}$ cancer-preventing, ${ }^{12}$ and liverprotective. ${ }^{13}$ Compared with garlic powder and garlic oil, AGE has been shown to be the most useful and effective garlic product $^{\mathbf{1 4}}$ as antioxidant in medicine due to its powerful antioxidant and free radical scavenging properties. ${ }^{15}$ In our previous work, ${ }^{16,17}$ similar results were obtained, the AGE showed strong vitro antioxidant activity (analyzed by 2,2-diphenyl-1picrylhydrazyl (DPPH) assay, 2,2'-azinobis-(3ethylbenzothiazoline-6-sulfonate) (ABTS) assay, hydroxyl free radicals assay and ferric reducing/antioxidant power (FRAP) assay). The bioactive ingredients of AGE including organosulfur compounds, saponins, flavonoids and phenols were identified by LC-MS/MS and GC-MS. Besides some well-known organosulfur compounds, other substances such as $S$-allylcysteine, $S$ allylmercaptocysteine,$^{18}$ the $\gamma$-glutamyl tripeptides ${ }^{19}$ and tetrahydro- $\beta$-carboline derivatives ${ }^{\mathbf{2 0}}$ were identified in AGE as potent antioxidants.

A large number of studies have proved that AGE has positive therapeutic and preventive effects on diabetes, ${ }^{21}$ cardiovascular diseases, ${ }^{22}$ neurodegenerative diseases ${ }^{23}$ and hyperlipidemia ${ }^{24}$ through its antioxidant properties. The mechanism and metabolic pathway of AGE on these diseases had also been separately studied. However, the metabolic pathways associated with the antioxidant function of AGE had not been fully systematically studied. Different from the previous reported literatures, we tried to make up for the research gap between metabolic 
Table 1 Effects of AGE on the content of MDA and lipofuscin in liver, brain of D-galactose-induced mice $(\text { mean } \pm S D, n=12)^{a}$

\begin{tabular}{|c|c|c|c|c|}
\hline \multirow[b]{2}{*}{ Groups } & \multicolumn{2}{|l|}{ MDA } & \multicolumn{2}{|l|}{ Lipofuscin } \\
\hline & $\begin{array}{l}\text { Brain (nmol } \\
\mathrm{mg}^{-1} \text { pro) }\end{array}$ & Liver (nmol mg ${ }^{-1}$ pro) & Brain $\left(n g \mathrm{~g}^{-1}\right)$ & Liver $\left(n g \mathrm{~g}^{-1}\right)$ \\
\hline $\mathrm{N}$ & $3.35 \pm 0.12$ & $2.47 \pm 0.11$ & $41.12 \pm 1.65$ & $35.63 \pm 3.29$ \\
\hline $\mathrm{C}$ & $6.69 \pm 0.44^{\# \#}$ & $2.78 \pm 0.16^{\# \#}$ & $49.08 \pm 1.73^{\# \#}$ & $57.16 \pm 4.99^{\# \#}$ \\
\hline L-1 & $3.96 \pm 0.15^{* *}$ & $2.57 \pm 0.08$ & $50.53 \pm 3.44$ & $58.80 \pm 3.77$ \\
\hline L-2 & $4.46 \pm 0.60^{* *}$ & $2.19 \pm 0.07^{* *}$ & $47.31 \pm 3.41$ & $57.40 \pm 2.14$ \\
\hline M-2 & $2.79 \pm 0.23^{* *}$ & $2.10 \pm 0.20^{* *}$ & $49.23 \pm 3.45$ & $56.85 \pm 4.57$ \\
\hline $\mathrm{H}-2$ & $3.69 \pm 0.11^{* *}$ & $2.24 \pm 0.15^{* *}$ & $36.56 \pm 5.10^{* *}$ & $43.95 \pm 3.54^{* *}$ \\
\hline
\end{tabular}

pathways and the antioxidant function of AGE, and link the antioxidant function of AGE to the metabolic pathways. Furthermore, it may link the metabolic pathways affected by AGE with aging hallmarks, and laying the foundation for studying the anti-aging mechanism of AGE.

D-Galactose-induced mice been used as an animal model of oxidative damage. ${ }^{25}$ Rodents injected with D-galactose display symptoms which resemble accelerated aging. The free radicals generated from oxidation of D-galactose overrun the capacity of cells to neutralize them. This would cause the chain reaction of lipid peroxidation and the result of end products, such as malondialdehyde (MDA), which could be combined with protein and phospholipid, leading to injury of the cellular membrane. ${ }^{26}$ In addition, accumulation of lipofuscin could damage nerves and lead to brain dysfunction. Numerous studies reported that the accumulation of lipofuscin could also cause damage to proteins, DNA replication and lead to RNA synthesis blocked dysfunction. ${ }^{27}$ The long-term administration of D-galactose could induce changes in these redox-related biomarkers in mice, including decreasing in total superoxide dismutase (T-SOD), glutathione peroxidase (GSH-Px), and catalase (CAT) activities, as well as increasing of the MDA and lipofuscin level.

In the present study, the vivo antioxidant activity of two selfmade AGE was explored through the treatment effects of the self-made AGE to D-galactose-induced mice. To study vivo antioxidant activity of AGE, some important index of antioxidant activity, such as the levels of T-SOD, GSH-Px, CAT activity in serum, brain and liver, and the content of MDA and lipofuscin in brain and liver were measured. And the brain and liver tissue were observed by paraffin section, the size, shape and arrangement state of mouse brain and liver cells were analyzed. Subsequently, gene expression profile array was used to analyze the influence of the self-made AGE on gene expression. To prove the correctness of the differentially expressed genes obtained through cDNA microarray analysis, PCR amplification was applied to verify the randomly selected differentially expressed genes. The differentially expressed genes screened through gene expression profile array were used to find the related metabolic pathways with the GenBank ID in the NCBI gene database and KEGG metabolic pathway database. Furthermore, the vivo antioxidant mechanisms of AGE were analyzed. Therefore, the objective of the present study was to research the vivo antioxidant mechanisms of the two self-made AGE on Dgalactose-induced mice and investigate the relationship between the antioxidant function of AGE and the metabolic pathways.

\section{Materials and methods}

\subsection{Chemicals and animals}

Fresh garlic was purchased from local markets (Tianjin, China). A total amount of 120 male ICR mice weighing $20 \pm 2 \mathrm{~g}$ (after adaption period) were purchased from Chinese People's Liberation Army Military Academy of Medical Sciences Laboratory Animal Center, license number SCXK (Beijing) 2012-0004.

\subsection{Preparation of two self-made AGE}

In order to obtain AGE with higher antioxidant activity, different conditions which might mainly affect the antioxidant of AGE, such as temperature, soaking time, soaking solution and the ratio of solid to liquid, were considered as main factors to be investigated detailed in the experiment. Sliced fresh garlic were soaked in different concentrations of ethanol solution $(0 \%$, $10 \%, 20 \%, 30 \%$ ) and stored in the dark, the antioxidant activity (analyzed by DPPH, ABTS, hydroxyl free radicals and FRAP assay) of the extract were monitored every ten days until the 210th day. According to the results of antioxidant activity changing with time from 0 to 210th day, we obtained two optimal production process of AGE, the AGE-1 was prepared in $10 \%$ ethanol for 90 days with soaking temperature of $25{ }^{\circ} \mathrm{C}$ and solid to liquid ratio of $1: 6 ;^{16}$ the AGE- 2 was prepared in distilled water for 20 days with soaking temperature of $25{ }^{\circ} \mathrm{C}$ and solid to liquid ratio of $1: 6,{ }^{17}$ and then the extract were filtered and concentrated under reduced pressure at $40{ }^{\circ} \mathrm{C}$. The antioxidant activity of the AGE-1 and AGE- 2 were analyzed by DPPH, ABTS assay, the $\mathrm{EC}_{50}\left(\mathrm{mg} \mathrm{mL}{ }^{-1}\right.$, dry weight) of the AGE-1 on ABTS assay and DPPH assay were 45.8 and 48.7 , respectively; the $\mathrm{EC}_{50}$ (mg mL $\mathrm{mL}^{-1}$, dry weight) of the AGE-2 on ABTS assay and DPPH assay were 27.6 and 36.9, respectively. AGE-1 and AGE-2 were 
prepared into the required concentration using sterile physiological saline for animal experiments.

\subsection{Treatments and statement of ethical animal treatment procedures}

All the experimental procedures were in accordance with the Committee on the Ethics of Animal Experiments of Tianjin University of Science \& Technology, and were based on the National Institutes of Health Guide for Care and Use of Laboratory Animals. Five mice per cage were randomly divided and housed under standard conditions at $20-25{ }^{\circ} \mathrm{C}$, a $12 \mathrm{~h}$ light/dark cycle and a relative humidity of $45 \%-65 \%$ with food and water available and libitum during the adaption period. After 7 days of adaption period, the mice were randomly divided into normal group, control group, AGE-1 and AGE-2 test group. Each treatment group was divided into three doses of high, middle and low groups. These groups were given normal diet and referred as N, C, L-1, M-1, H-1, L-2, M-2, and $\mathrm{H}-2$ groups, respectively. Since the start of the experiment, mice in the $\mathrm{N}$ group were i.h. injected with physiological saline and p.o. administered with physiological saline. Mice in the $\mathrm{C}$ group were i.h. injected with D-galactose $(120 \mathrm{mg}$ per $\mathrm{kg}$ bw per d) and p.o. administered with physiological saline. Mice in L-1, M-1, H-1 groups were i.h. injected with D-galactose (120 mg per $\mathrm{kg}$ bw per d) and p.o. administered with AGE-1 (1, 2 and $4 \mathrm{~g}$ per $\mathrm{kg}$ bw per d). Mice in L-2, M-2, and $\mathrm{H}-2$ groups were i.h. injected with $\mathrm{D}$-galactose (120 $\mathrm{mg}$ per $\mathrm{kg}$ bw per d) and p.o. administered with AGE-2 (1, 2 and $4 \mathrm{~g}$ per kg bw per d). The experiment was carried out continuously for eight weeks.

After eight weeks experiment, all mice were fasted overnight. After the blood was collected by the eyeball removal, the mice were sacrificed by cervical vertebra dislocation. The blood samples were centrifuged at $3000 \mathrm{rpm}$ for $10 \mathrm{~min}$ at $4{ }^{\circ} \mathrm{C}$, and the serums were separated and stored at $-80{ }^{\circ} \mathrm{C}$ until biochemical analysis. The liver and brain were harvested, rinsed with cold normal saline, and then weighed. Subsequently, 10\% (w/v) liver and brain tissue homogenate were prepared in normal saline using a glass homogenizer. The homogenate was centrifuged twice at $4000 \mathrm{rpm}$ for $10 \mathrm{~min}$ at $4{ }^{\circ} \mathrm{C}$, and the supernatant was used for biochemical assays. Portions of the liver and brain tissues were immersed in $10 \%$ neutral formalin solution for histopathological observation. The remaining liver and brain tissues were immediately frozen in liquid nitrogen and stored at $-80{ }^{\circ} \mathrm{C}$ until use for gene chip analysis.

\subsection{Biochemical analysis in serum, brain and liver}

The levels of T-SOD, GSH-Px, CAT in serum, brain and liver, and the content of MDA in brain and liver were measured according to the manufacturers' instructions of the commercially available kits. In addition, the level of lipofuscin in brain and liver was determined by enzyme linked immunosorbent assay (ELISA). The total protein content in the liver and brain tissue homogenate was determined using the Coomassie brilliant blue method following the manufacturer's instruction. 

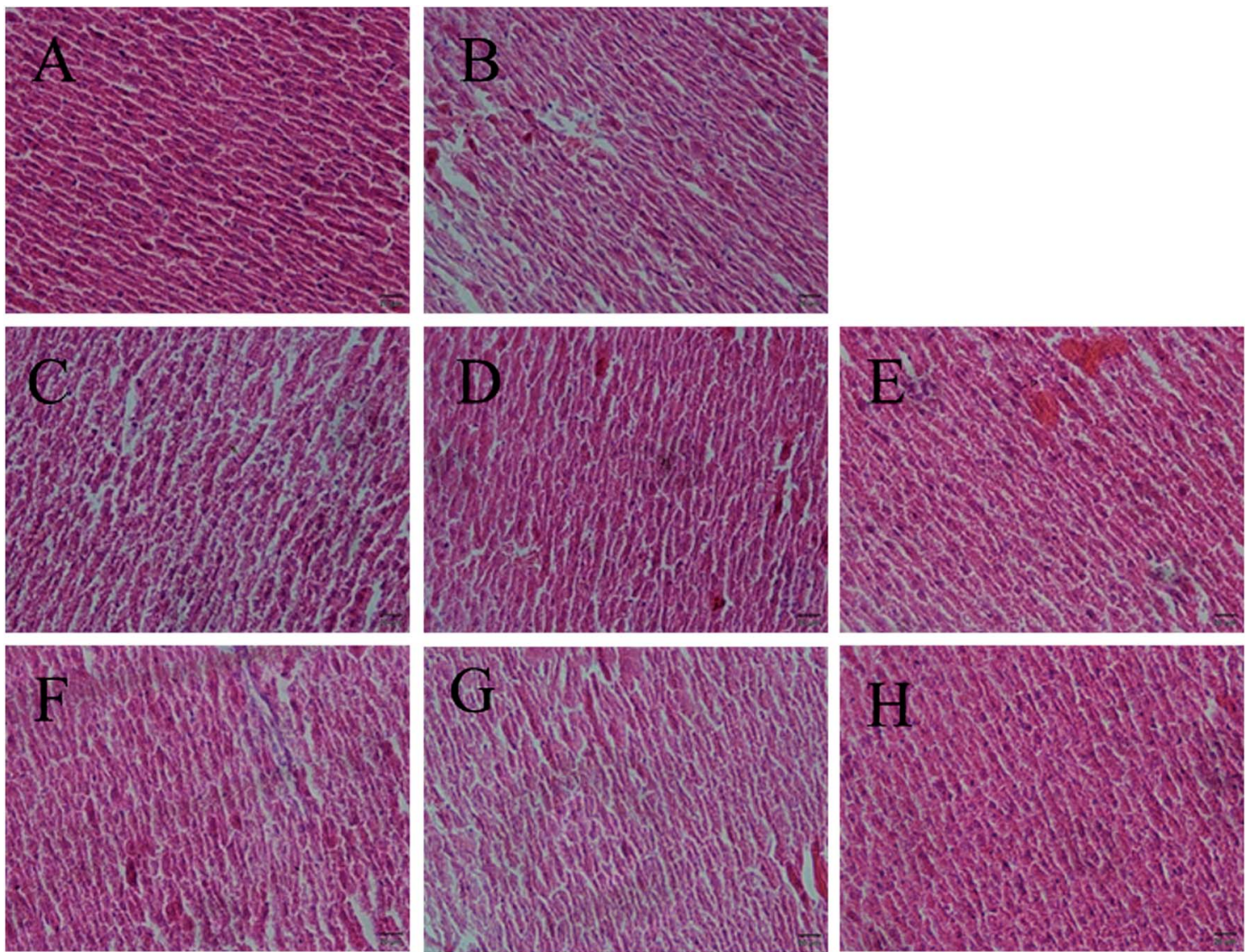

Fig. 1 Histopathological analysis of liver. All pictures were taken at 200 times. The letters $(\mathrm{A}-\mathrm{H})$ among the figure represent in proper order following groups. $\mathrm{N}, \mathrm{C}, \mathrm{L}-1, \mathrm{M}-1, \mathrm{H}-1, \mathrm{~L}-2, \mathrm{M}-2$, and $\mathrm{H}-2$ groups.

\subsection{Histological analysis}

Liver and brain tissues were fixed in formalin solution, dehydrated with ethanol, embedded with paraffin, serially sectioned and stained with hematoxylin and eosin. The above prepared samples were then observed microscopically.

\subsection{Gene chip analysis, RT-PCR verification, and metabolic pathway analysis}

Differentially expressed genes were screened using gene expression profile array (GeneChip Mice 430 plus 2.0 Array, Affymetrix, USA). First, liver and brain tissues were ground in liquid nitrogen. Total RNA was extracted using Trizol and then purified in an RNeasy column (RNeasy mini kit, Qiagen, Düsseldorf, North Rhine-Westphalia, Germany). Then, cDNA was synthesized by reverse transcription from total RNA, amplified through in vitro transcription reaction (IVT) using the 3' IVT PLUS Reagent Kit (Affymetrix), and then purified with purification beads. The purified cRNA was fragmented by divalent cations and elevated temperature. Finally, the hybridized array of cRNA was placed in a hybridization oven, washed, stained, and then scanned with the help of biotin and fluorescence labels used during cRNA synthesis and staining. Optical signals were converted to electrical signals and turn electrical signals into raw data. After analysis, the expression data were used to screen the co-expressed genes in accordance with the following condition: the expression value difference of the genes in $\mathrm{N}, \mathrm{H}-1$, and $\mathrm{M}-2$ group should be more than 100 and two-fold change compared with $\mathrm{C}$ group.

The differentially expressed genes screened by gene chip arrays were verified through RT-PCR. Specific primers for the genes were designed, synthesized, and diluted with sterile water in accordance with the manufacturer's manual. The total RNA in the liver and brain tissues served as the raw material, whereas the mice $\beta$-actin genes were used as house-keeping genes. The One-Step SYBR PrimeScript PLUS RT-PCR kit (TaKaRa, Japan) was used for PCR reaction.

The differentially expressed genes screened through gene expression profile array were used to find the related metabolic pathways with the GenBank ID in the NCBI gene database and KEGG metabolic pathway database. The effects of the resulting genes on the metabolic pathway were also explored.

\subsection{Statistical analysis}

The data are represented as mean $\pm \mathrm{SD}$. All data were analyzed by one-way analysis of variance (ANOVA), and Duncan's multiple range tests were performed to determine the significant differences between groups using SPSS software (Chicago, IL, USA). 

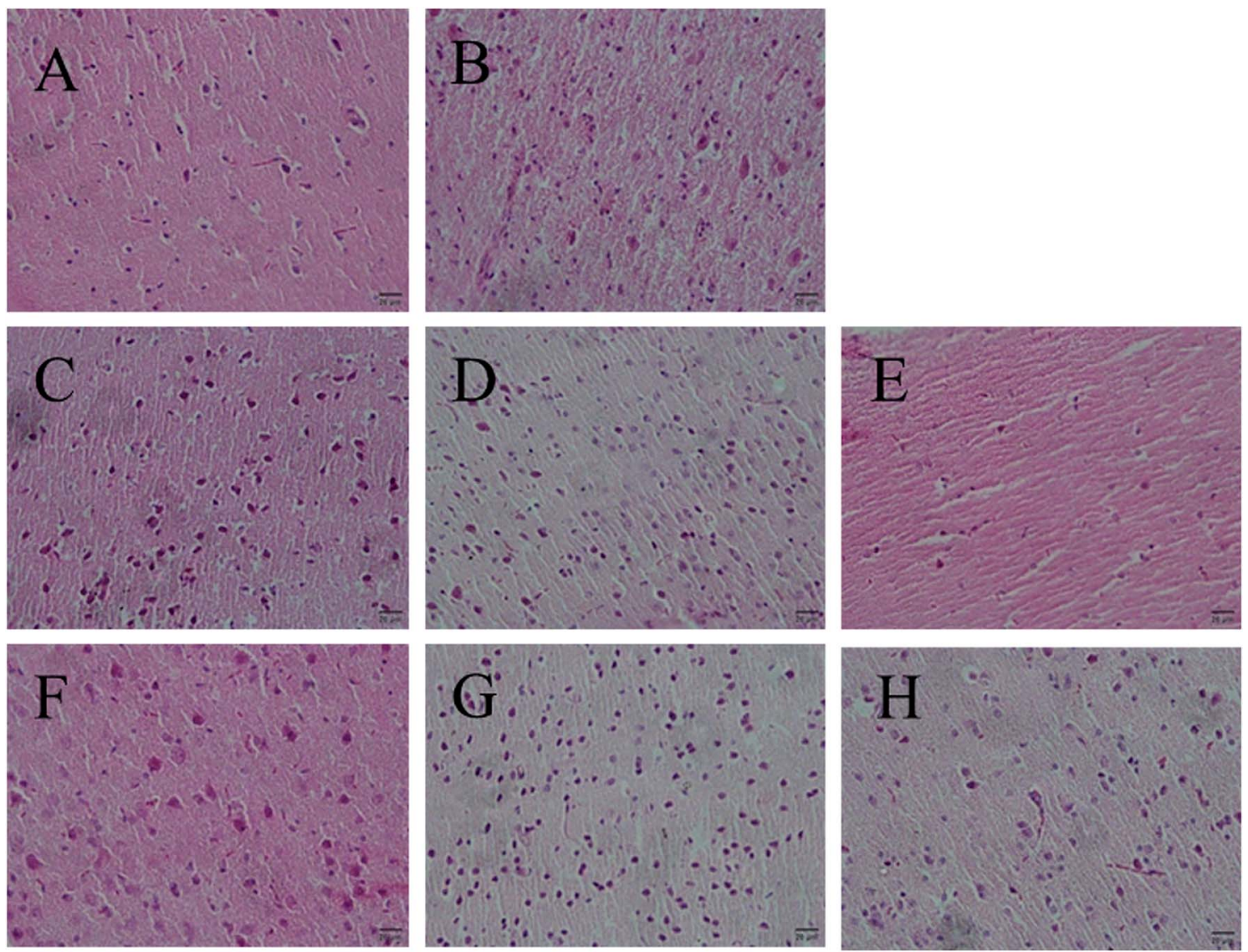

Fig. 2 Histopathological analysis of brain. All pictures were taken at 200 times. The letters $(\mathrm{A}-\mathrm{H})$ among the figure represent in proper order following groups, $N, C, L-1, M-1, H-1, L-2, M-2$, and $H-2$ groups.

\section{Results}

\subsection{Biochemical analysis results}

The levels of MDA and lipofuscin in brain and liver from different groups were shown in Table 1. In comparison with mice in $\mathrm{N}$ group, the levels of MDA and lipofuscin of $\mathrm{C}$ group in both brain and liver significantly increased. The levels of MDA in both brain and liver of mice in $\mathrm{H}-1, \mathrm{M}-2$ and $\mathrm{H}-2$ group were significantly lower than that of $\mathrm{C}$ group. Meanwhile, the levels of lipofuscin of mice in $\mathrm{H}-1$ and $\mathrm{H}-2$ group in both brain and liver were significantly lower than that of $\mathrm{C}$ group.

Antioxidant enzyme activities in serum, brain and liver were shown in Table 2. In comparison to mice in $\mathrm{N}$ group, the levels of T-SOD, GSH-Px, and CAT of C group in both serum, brain and liver were significantly decreased, which indicated that the model was successfully established. The levels of GSH-px and CAT of M-1 and H-1 group in both serum, brain and liver were significantly higher than that of $\mathrm{C}$ group. Compared with $\mathrm{C}$ group, the levels of T-SOD of M-2 and $\mathrm{H}-2$ group in both serum, brain and liver were significantly increased. From the results of important indicators, the high dose of AGE-1 (H-1) and the middle dose of AGE-2 (M-2) showed strong vivo antioxidant activities.

Free radicals and oxidants can affect the body's metabolic function, causing various health problems. Eliminating excessive oxidative free radicals can prevent many diseases caused by free radicals and diseases related to aging. The self-made AGE-1 and AGE- 2 could significantly reduce the oxidation of body load, reduce lipid peroxidation and thus were able to exert its resistance through the increasing of T-SOD, GSH-Px and CAT activity with the decreasing of MDA and lipofuscin content.

\subsection{Tissue morphology}

Antioxidants could preserve tissue morphology from oxidative damages induced by D-galactose. Thus, different dose groups of AGE-1 and AGE-2 effects on liver and brain tissue of D-galactoseinduced mice were observed. The histology results of mice liver and brain tissue were shown in Fig. 1 and 2. Liver tissue cells of mice in $\mathrm{N}$ group with uniform size, normal structure, small cell gap were easily observed and the nucleus were clearly visible. Compared with $\mathrm{N}$ group, liver tissue cells of mice in $\mathrm{C}$ group were swelling and inconsistent, which were arranged in loose disorder and the nucleus were not obvious. The high, middle and low doses of AGE-1 and AGE- 2 could improve the size, shape and arrangement state of liver cells with dose-dependent relationships. Wherein, liver tissues of $\mathrm{H}-2$ group were closed to the state of normal group to some extent. Brain tissue cells of mice in $\mathrm{N}$ group were arranged neatly, tight and regular. As shown in the $\mathrm{C}$ group, long-term injection of D-galactose could cause loose disordered arrangement of brain cells. As indicated in the Fig. 2, AGE-1 and AGE-2 in low, middle and high dose 
Table 3 Co-expression of down-regulated genes and up-regulated genes in liver tissues

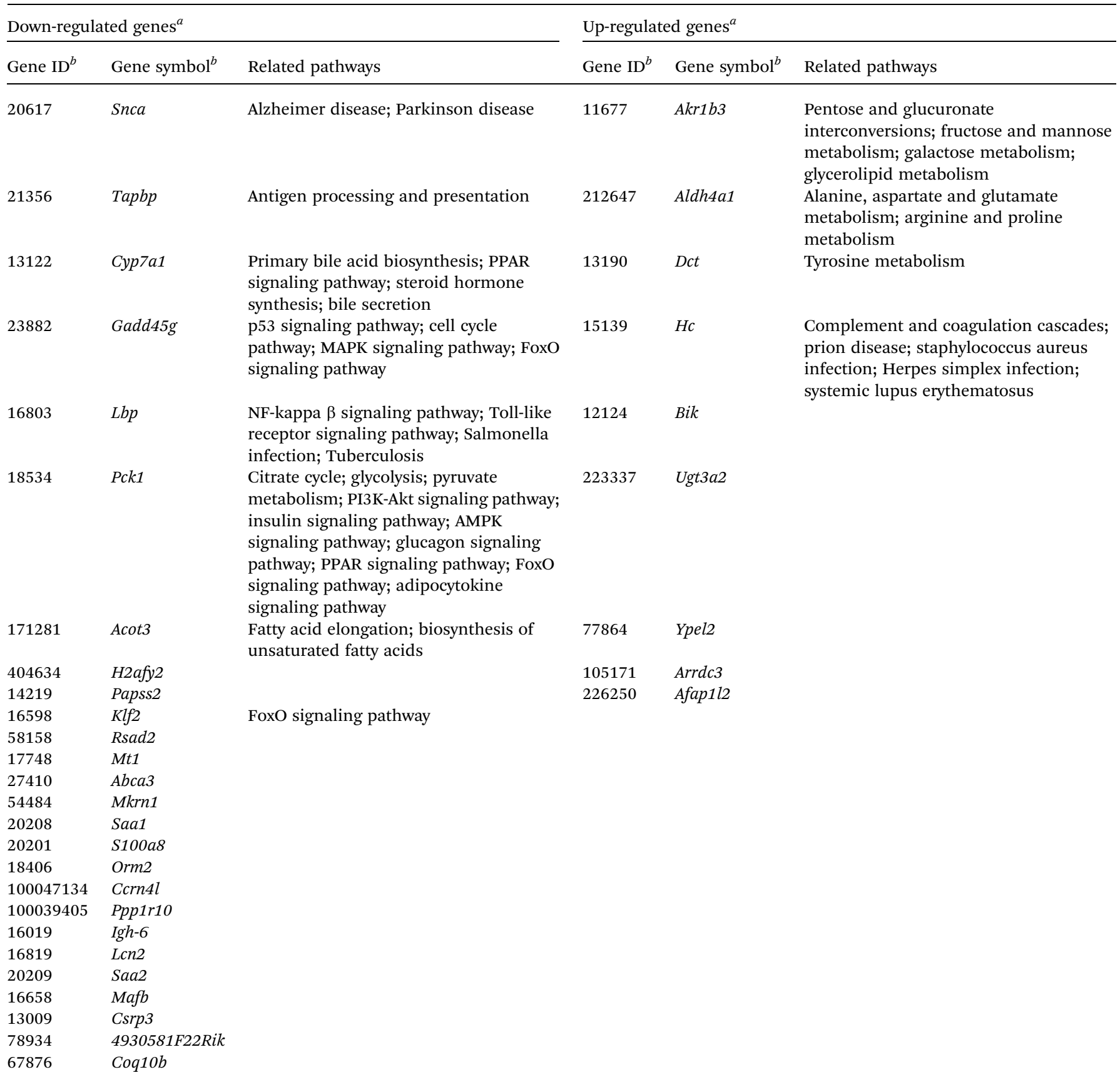

${ }^{a}$ The expression value difference of the genes in $\mathrm{N}, \mathrm{H}-1$, and $\mathrm{M}-2$ group should be more than 100 and two-fold change compared with $\mathrm{C}$ group. ${ }^{b}$ National Center for Biotechnology Information (NCBI) Entrez Gene.

groups could all improve the size, shape and arrangement state of brain cells. Generally, AGE-1 and AGE-2 in low, middle and high dose groups could all improve the size, shape and arrangement state of liver and brain cells, which was also benefit from AGE's powerful antioxidant activity.

\subsection{Differentially expressed genes and metabolic pathway}

Gene expression profile array is one of the most important methods of current research to explore the molecular mechanism between nutrients and chronic diseases. By comparing the changes in gene expression profiles and related metabolic pathways, the target of the sample can be directly searched at the gene level to explain its mechanism of action. To further study the vivo antioxidant mechanism of self-made AGE on the D-galactose-induced mice, gene expression profile array was conducted to screen differentially expressed genes in liver and brain, the results were shown in Table 3 and 4 .

Compared with $\mathrm{C}$ group, 35 differentially co-expressed genes in liver of mice in N, H-1 and M-2 group were found. Within the 35 genes, there were 9 genes $(A k r 1 b 3$, Aldh4a1, Hc, Dct, Bik, Ugt3a2, Ypel2, Arrdc3, Afap1l2) significantly up-regulated and 26 genes (Snca, Tapbp, Cyp7a1, Gadd45g, Lbp, Pck1, Acot3, H2afy2, 
Table 4 Co-expression of down-regulated genes and up-regulated genes in brain tissues

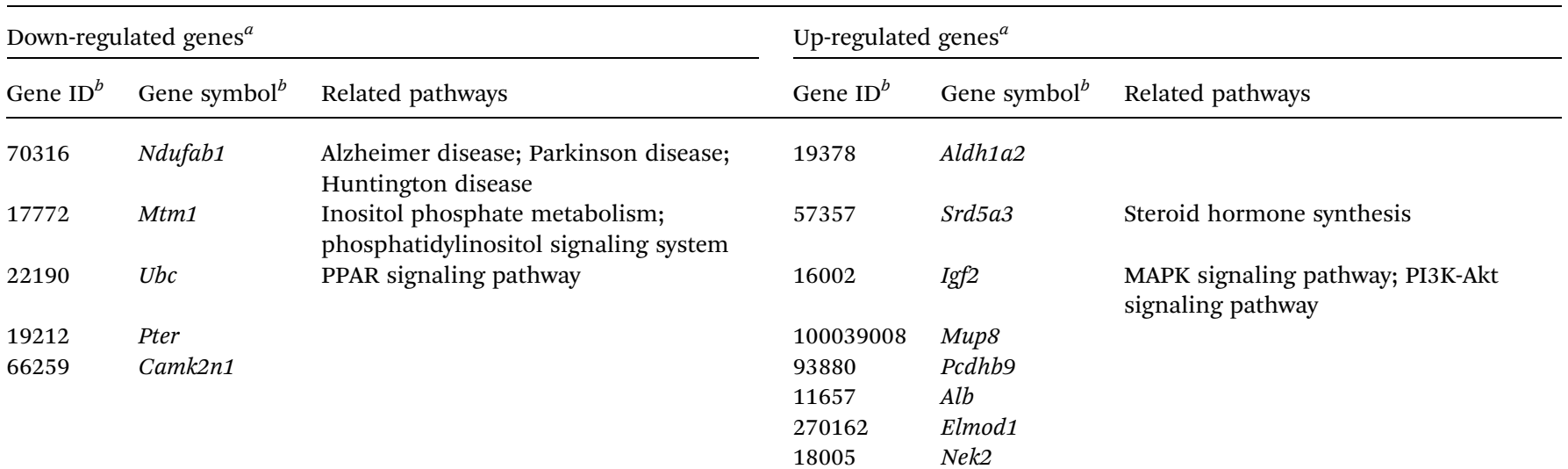

${ }^{a}$ The expression value difference of the genes in N, H-1, and M-2 group should be more than 100 and two-fold change compared with C group.

${ }^{b}$ National Center for Biotechnology Information (NCBI) Entrez Gene.

Papss2, Klf2, Rsad2, Mt1, Abca3, Mkrn1, Saa1, S100a8, Orm2, Ccrn4l, Ppp1r10, Igh-6, Lcn2, Saa2, Mafb, Csrp3, F22Rik, Coq10b) significantly down-regulated (Table 3). 13 differentially coexpressed genes in brain of mice in $\mathrm{N}, \mathrm{H}-1$ and $\mathrm{M}-2$ group were screened by gene expression profile array. Within the 13 genes, there were 8 genes (Aldh1a2, Srd5a3, Igf2, Mup8, Pcdhb9, $A l b$, Elmod1, Nek2) significantly up-regulated and 5 genes (Ndufab1, Mtm1, Ubc, Pter, Camk2n1) significantly downregulated (Table 4).

Specific primers were used for the PCR amplification of the 8 randomly selected differentially expressed genes (Snca, Pck1, Ndufab1, Mtm1, Akr1b3, Dct, Srd5a3, Aldh1a2), with the housekeeping gene $\beta$-actin as the internal standard and the RNA in C group as the control (Fig. 3). The results were relatively quantified using the $2^{-\Delta \Delta C_{\mathrm{t}}}$ method, which proved the correctness of the differentially expressed genes obtained through cDNA microarray analysis.

Then these differentially expressed genes listed in Tables 3 and 4 were search on KEGG database to analyze the influence of the self-made AGE on gene expression and metabolic pathways of mice, and 41 metabolic pathways have been discovered, including 10 carbohydrate metabolism pathways (citrate cycle pathway, glycolysis pathway, pyruvate metabolism pathway, PI3K-Akt signaling pathway, insulin signaling pathway, AMPK signaling pathway, glucagon signaling pathway, pentose and glucuronate interconversions pathway, fructose and mannose metabolism pathway, galactose metabolism pathway), 11 immune response pathways (NF-kappa $\beta$ signaling pathway, Toll-like receptor signaling pathway, antigen processing and presentation pathway, Salmonella infection pathway, systemic lupus erythematosus pathway, prion disease pathway, Tuberculosis pathway, complement and coagulation cascades pathway, Herpes simplex infection pathway, pertussis pathway, Staphylococcus aureus infection pathway), 8 lipid metabolism pathways (fatty acid elongation pathway, primary bile acid biosynthesis pathway, biosynthesis of unsaturated fatty acids pathway, adipocytokine signaling pathway, glycerolipid metabolism pathway, PPAR signaling pathway, steroid hormone synthesis pathway, bile secretion pathway), 6 cell cycle pathways (inositol phosphate metabolism pathway, phosphatidylinositol signaling system pathway, p53 signaling pathway, cell cycle pathway, MAPK signaling pathway, FoxO signaling pathway), 3 amino acid metabolism pathways (tyrosine metabolism pathway, arginine and proline metabolism pathway, alanine,
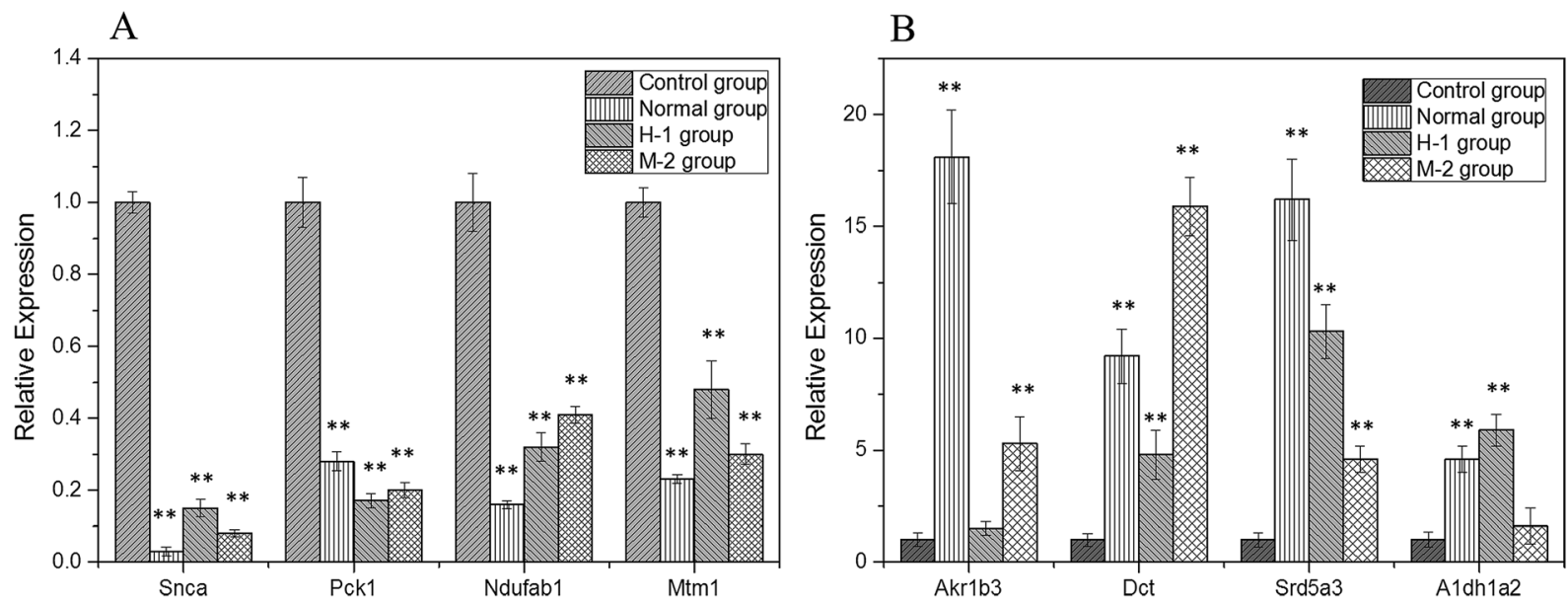

Fig. 3 The RNA relative expression levels of down-regulated (A) and up-regulated genes (B). Compared with $C$ group, ** $P<0.01$. 
aspartate and glutamate metabolism pathway) and 3 nervous system diseases pathways (Alzheimer disease pathway, Parkinson disease pathway, Huntington disease pathway). These results indicated that the differentially expressed genes played important roles in carbohydrate metabolism, lipid metabolism, amino acid metabolism, immune response, cell cycle, and nervous system regulation.

\section{Discussion}

Carlos López-Otín et al. ${ }^{28}$ reported nine hallmarks which represent common denominators of aging in different organisms, these hallmarks were: genomic instability, telomere attrition, epigenetic alterations, loss of proteostasis, deregulated nutrient sensing, mitochondrial dysfunction, cellular senescence, stem cell exhaustion, and altered intercellular communication. Among these metabolic pathways that have been affected by AGE- 1 and AGE- 2 in the D-galactose-caused injury model, NF-kappa $\beta$ signaling pathway was associated with the altered intercellular communication, epigenetic alterations, and genomic instability; ${ }^{29-31}$ p53 signaling pathway was related to cellular senescence and genomic instability; ${ }^{32,33}$ FoxO signaling pathway, insulin signaling pathway, PI3K-Akt signaling pathway and AMPK signaling pathway could regulated the nutrient sensing; ${ }^{34-38}$ citrate cycle pathway could affect the mitochondrial function; ${ }^{39}$ Alzheimer disease pathway and Parkinson disease pathway was related to loss of proteostasis. ${ }^{\mathbf{4 0}}$ These results indicated that AGE- 1 and AGE- 2 could prevent the mice from aging by regulated genomic stability, epigenetics, proteostasis, nutrient sensing, mitochondrial function, cellular senescence, and intercellular communication.

An activity guided fractionation approach were conducted to guide the fractionation by means of extraction, column chromatography and semi-preparative HPLC in previous research of our group. ${ }^{17}$ The tetrahydro- $\beta$-carboline derivatives were separated and identified as main antioxidants in AGE-1 and AGE-2 by IR, UV, MS, ${ }^{1} \mathrm{H}$ NMR, ${ }^{13} \mathrm{C}$ NMR spectrometry, and the content of tetrahydro- $\beta$-carboline derivatives in AGE- 1 and AGE2 were $0.93 \mathrm{~g} \mathrm{~kg}^{-1}$ and $0.54 \mathrm{~g} \mathrm{~kg}^{-1}$ dry AGE, respectively. Another major active antioxidant $S$-allylcysteine content were $1.6 \mathrm{~g} \mathrm{~kg}^{-1}$ and $1.8 \mathrm{~g} \mathrm{~kg}^{-1}$ (dry weight), in AGE-1 and AGE-2, respectively. $S$-Allylcysteine was reported as the main effective antioxidants in AGE, which could inhibit free radical production, lipid peroxidation and neuronal damage. ${ }^{41}$ In this experiment, 10 carbohydrate metabolism pathways were affected by AGE-1 and AGE-2, this might be related to the biological activity of $S$-allylcysteine, which was reported to prevent formation of advanced glycation endproducts. ${ }^{42}$ In addition, $S$ allylcysteine could protect against oxidative stress in Parkinsonism by neuromodulation and immunomodulatory. ${ }^{43,44}$ In the present experiment, AGE-1 and AGE-2 participated in Parkinson disease pathway, this may be due to the contribution of $S$-allylcysteine in AGE-1 and AGE-2. According to the $\mathrm{EC}_{50}$ value on DPPH assay and the content of tetrahydro- $\beta$-carboline derivatives in the AGE- 1 and AGE-2, the antioxidant activities contribution of tetrahydro- $\beta$-carboline derivatives in AGE- 1 and AGE-2 was 4.3 and 2.2 times towards that of $S$-allylcysteine, respectively. ${ }^{17}$ So the tetrahydro- $\beta$-carboline derivatives mainly contribute to the potent antioxidant properties of AGE- 1 and AGE-2.

\section{Conclusion}

With the aging acceleration of social population, the efficacy and mechanism of AGE, as a promising garlic product, in many biofunctional aspects need to be explored. In conclusion, longterm injection of D-galactose could accelerate the ageing of mice, AGE- 1 and AGE- 2 could significantly reduce the oxidation of body load, reduce lipid peroxidation and thus were able to exert its resistance through the increasing of T-SOD, GSH-Px and CAT activity with the decreasing of MDA and lipofuscin content. Results of gene expression profile array showed that the two self-made AGE could protect the mice from D-galactosecaused injury by multiple aspects, such as carbohydrate metabolism, immunomodulatory, lipid metabolism, cell cycle regulation, amino acid metabolism and nervous regulation pathways. Further mechanism analysis found that AGE-1 and AGE- 2 could prevent the mice from aging by regulated genomic stability, epigenetics, proteostasis, nutrient sensing, mitochondrial function, cellular senescence, and intercellular communication. Through this experiment, we had comprehensively study the antioxidant mechanism of AGE in vivo and linked the antioxidant function of AGE to the metabolic pathways. Meanwhile, the research results of this experiment could provide guidance for further anti-aging research of AGE.

\section{Conflict of interest}

The authors declare no conflicts of interest.

\section{Acknowledgements}

The research was supported by Shanxi applied basic research project (201601D021116, 201701D221180), and the National Science Foundation of China (project No. 31701686), Scientific and Technological Innovation Programs of Higher Education Institutions in Shanxi (project No. 201802016), and Shanxi University of Chinese Medicine Doctoral Research Fund (2016BK11).

\section{References}

1 D. Harman, Aging: a theory based on free radical and radiation chemistry, J. Gerontol., 1956, 11, 298-300.

2 B. Halliwell, Free radicals and antioxidants: updating a personal view, Nutr. Rev., 2012, 70, 257-265.

3 N. Houstis, E. D. Rosen and E. S. Lander, Reactive oxygen species have a causal role in multiple forms of insulin resistance, Nature, 2006, 440, 944-948.

4 D. Milatovic, S. Zaja-Milatovic, R. C. Gupta, Y. Yu and M. Aschner, Oxidative damage and neurodegeneration in manganese-induced neurotoxicity, Toxicol. Appl. Pharmacol., 2009, 240, 219-225. 
5 J. G. Park and G. T. Oh, The role of peroxidases in the pathogenesis of atherosclerosis, BMB Rep., 2011, 44, 497505.

6 H. C. Lee and Y. H. Wei, Oxidative stress, mitochondrial DNA mutation, and apoptosis in aging, Exp. Biol. Med., 2007, 232, 592-606.

7 H. Amagase, B. L. Petesch, H. Matsuura, S. Kasuga and Y. Itakura, Intake of garlic and its bioactive components, $J$. Nutr., 2001, 131, 955S-962S.

8 Y. Kodera, A. Suzuki, O. Imada, S. Kasuga, I. Sumioka, A. Kanezawa, N. Taru, M. Fujikawa, S. Nagae, K. Masamoto, K. Maeshige and K. Ono, Physical, chemical, and biological properties of s-allylcysteine, an amino acid derived from garlic, J. Agric. Food Chem., 2002, 50, 622-632.

9 N. Morihara, A. Hino, S. Miki, M. Takashima and J. I. Suzuki, Aged garlic extract suppresses inflammation in apolipoprotein E-knockout mice, Mol. Nutr. Food Res., 2017, 61, 1700308.

10 S. S. Percival, Aged Garlic Extract Modifies Human Immunity, J. Nutr., 2016, 146, 433S-436S.

11 M. Ushijima, M. Takashima, K. Kunimura, Y. Kodera, N. Morihara and K. Tamura, Effects of S-1-propenylcysteine, a sulfur compound in aged garlic extract, on blood pressure and peripheral circulation in spontaneously hypertensive rats, J. Pharm. Pharmacol., 2018, 70, 559-565.

12 S. Tanaka, K. Haruma, M. Yoshihara, G. Kajiyama, K. Kira, H. Amagase and K. Chayama, Aged garlic extract has potential suppressive effect on colorectal adenomas in humans, J. Nutr., 2006, 136, 821S-826S.

13 S. Nakagawat, S. Kasuga and H. Matsuura, Prevention of liver damage by aged garlic extract and its components in mice, Phytother. Res., 2010, 3, 50-53.

14 S. Kasuga, N. Uda, E. Kyo, M. Ushijima, N. Morihara and Y. Itakura, Pharmacologic activities of aged garlic extract in comparison with other garlic preparations, J. Nutr., 2001, 131, 1080S-1084S.

15 N. Morihara and H. Fujii, Aged Garlic Extract Scavenges Superoxide Radicals, Plant Foods Hum. Nutr., 2011, 66, 1721.

16 X. Wang, Y. Yang, R. Liu, Z. Zhou and M. Zhang, Identification of Antioxidants in Aged Garlic Extract by Gas Chromatography-Mass Spectrometry and Liquid Chromatography-Mass Spectrometry, Int. J. Food Prop., 2016, 19, 474-483.

17 X. Wang, R. Liu, Y. Yang and M. Zhang, Isolation, purification and identification of antioxidants in an aqueous aged garlic extract, Food Chem., 2015, 187, 37-43.

18 J. Imai, N. Ide, S. Nagae, T. Moriguchi, H. Matsuura and Y. Itakura, Antioxidant and radical scavenging effects of aged garlic extract and its constituents, Planta Med., 1994, 60, 417-420.

19 M. Nakamoto, T. Fujii, T. Matsutomo and Y. Kodera, Isolation and identification of three $\gamma$-glutamyl tripeptides and their putative production mechanism in aged garlic extract, J. Agric. Food Chem., 2018, 66, 2891-2899.
20 M. Ichikawa, K. Ryu, J. Yoshida, N. Ide, S. Yoshida, T. Sasaoka and S. I. Sumi, Antioxidant effects of tetrahydro- $\beta$-carboline derivatives identified in aged garlic extract, BioFactors, 2010, 16, 57-72.

21 A. D. Abdel-Mageid, M. E. S. Abou-Salem, N. M. H. A. Salaam and H. A. S. El-Garhy, The potential effect of garlic extract and curcumin nanoparticles against complication accompanied with experimentally induced diabetes in rats, Phytomedicine, 2018, 43, 126-134.

22 I. Pérez-Torres, J. C. Torres-Narváez, J. Pedraza-Chaverri, M. E. Rubio-Ruiz, E. Díaz-Díaz, V.-M. L. Del, R. MartínezMemije, L. E. Varela and V. Guarner-Lans, Effect of the Aged Garlic Extract on Cardiovascular Function in Metabolic Syndrome Rats, Molecules, 2016, 21, 1425.

23 Z. Qu, V. V. Mossine, J. Cui, G. Y. Sun and Z. Gu, Protective Effects of AGE and Its Components on Neuroinflammation and Neurodegeneration, NeuroMol. Med., 2016, 18, 474-482.

24 S. M. B. Asdaq, Antioxidant and Hypolipidemic Potential of Aged Garlic Extract and Its Constituent, S-Allyl Cysteine, in Rats, Evid.-Based Complementary Altern. Med., 2015, 2015, 328545.

25 J. Lu, Y. Zheng, D. Wu, L. Luo, D. Sun and Q. Shan, Ursolic acid ameliorates cognitiondeficits and attenuates oxidative damage in the brain of senescent mice induced by $\mathrm{D}$ galactose, Biochem. Pharmacol., 2007, 74, 1078-1090.

26 M. Hayakawa, K. Hattori, S. Sugiyama and T. Ozawa, Ageassociated oxygen damage and mutations in mitochondrial DNA in human hearts, Biochem. Biophys. Res. Commun., 1992, 189, 979-985.

27 U. T. Brunk and A. Terman, Lipofuscin: mechanisms of agerelated accumulation and influence on cell function, Free Radical Biol. Med., 2002, 33, 611-619.

28 C. López-Otín, M. A. Blasco, L. Partridge, M. Serrano and G. Kroemer, The hallmarks of aging, Cell, 2013, 153, 11941217.

29 A. Salminen, K. Kai and A. Kauppinen, Inflammaging: disturbed interplay between autophagy and inflammasomes, Aging, 2012, 4, 166-175.

30 Y. Kanfi, S. Naiman, G. Amir, V. Peshti, G. Zinman, L. Nahum, Z. Bar-Joseph and H. Y. Cohen, The sirtuin SIRT6 regulates lifespan in male mice, Nature, 2012, 483, 218-221.

31 G. Mariño, A. P. Ugalde, A. F. Fernández, F. G. Osorio, A. Fueyo, J. M. Freije and C. Lópezotín, Insulin-like growth factor 1 treatment extends longevity in a mouse model of human premature aging by restoring somatotroph axis function, Proc. Natl. Acad. Sci. U. S. A., 2010, 107, 1626816273.

32 M. Serrano, A. W. Lin, M. E. Mccurrach, D. Beach and S. W. Lowe, Oncogenic ras provokes premature cell senescence associated with accumulation of p53 and p16INK4a, Cell, 1997, 88, 593-602.

33 I. Varela, J. Cadiã Anos, A. M. PendÃ s, n. A. Gutiã RrezFernã, A. R. Folgueras, L. M. SÃ nchez, Z. Zhou, F. J. Rodrã-Guez, C. L. Stewart and J. A. Vega, Accelerated ageing in mice deficient in Zmpste24 protease is linked to p53 signalling activation, Nature, 2005, 437, 564-568. 
34 N. Barzilai, D. M. Huffman, R. H. Muzumdar and A. Bartke, The Critical Role of Metabolic Pathways in Aging, Diabetes, 2012, 61, 1315-1322.

35 L. C. Foukas, B. Bilanges, L. Bettedi, W. Pearce, K. Ali, S. Sancho, D. J. Withers and B. Vanhaesebroeck, Long-term p110 $\alpha$ PI3K inactivation exerts a beneficial effect on metabolism, EMBO Mol. Med., 2013, 5, 563-571.

36 O. Renner and A. Carnero, Mouse models to decipher the PI3K signaling network in human cancer, Curr. Mol. Med., 2009, 9, 612-625.

37 V. N. Anisimov, L. M. Berstein, I. G. Popovich, M. A. Zabezhinski, P. A. Egormin, T. S. Piskunova, A. V. Semenchenko, M. L. Tyndyk, M. N. Yurova and I. G. Kovalenko, If started early in life, metformin treatment increases life span and postpones tumors in female SHR mice, Aging, 2011, 3, 148-157.

38 C. J. Kenyon, The genetics of ageing, Nature, 2010, 464, 504512.

39 A. Giralt and F. Villarroya, SIRT3, a pivotal actor in mitochondrial functions: metabolism, cell death and aging, Biochem. J., 2012, 444, 1-10.

40 E. T. Powers, R. I. Morimoto, A. Dillin, J. W. Kelly and W. E. Balch, Biological and chemical approaches to diseases of proteostasis deficiency, Annu. Rev. Biochem., 2009, 78, 959-991.

41 Y. Numagami and S. T. Ohnishi, S-allylcysteine inhibits free radical production, lipid peroxidation and neuronal damage in rat brain ischemia, J. Nutr., 2001, 131, 1100S-1105S.

42 M. S. Ahmad, M. Pischetsrieder and N. Ahmed, Aged garlic extract and $\mathrm{S}$-allyl cysteine prevent formation of advanced glycation endproducts, Eur. J. Pharmacol., 2007, 561, 32-38.

43 J. C. Tobónvelasco, G. Vázquezvictorio, M. Macíassilva, E. Cuevas, S. F. Ali, P. D. Maldonado, M. E. Gonzáleztrujano, A. Cuadrado, J. Pedrazachaverrí and A. Santamaría, S-allyl cysteine protects against 6hydroxydopamine-induced neurotoxicity in the rat striatum: involvement of Nrf2 transcription factor activation and modulation of signaling kinase cascades, Free Radical Biol. Med., 2012, 53, 1024-1040.

44 P. Rojas, N. Serrano-García, O. N. Medina-Campos, J. Pedraza-Chaverri, P. D. Maldonado and E. Ruiz-Sánchez, S-Allylcysteine, a garlic compound, protects against oxidative stress in 1-methyl-4-phenylpyridinium-induced parkinsonism in mice, J. Nutr. Biochem., 2011, 22, 937-944. 